\title{
Research on performance Evaluation method to promote sustainable development of opening government affairs
}

\author{
Mingle Zhou ${ }^{1 *}$, Shengli Cao ${ }^{1}$, Ran Wang ${ }^{1}$ and Yu Wang ${ }^{1}$ \\ ${ }^{1}$ Qilu University of Technology (Shandong Academy of Sciences), Jinan, ShanDong 250000, China
}

\begin{abstract}
Open government affairs (OGA) play an important role in promoting national governance system and capacity. In order to realize an open and efficient government, it is necessary to scientifically evaluate the performance of government. The effect of OGA can be improved continuously through the feedback from evaluation, which is beneficial for the sustainable development of OGA. However, with the continuous development of OGA, the existing methods of evaluation are faced with such problems as poor-timeliness, high-cost and subjective uncertainty, which are difficult to satisfy the demands of performance evaluation of OGA. Therefore, this paper puts forward a performance evaluation method based on T-S fuzzy neural network. Our method has a strong ability of data processing, which can simplify the work flow. The T-S fuzzy neural network was trained and tested through using the performance evaluation data of all districts and counties in Shandong Province, China. Finally, the evaluation results offered by our method are highly accurate. Hence, our method is suitable for the performance evaluation of OGA, it can continuously enhance the improvement of government's performance management and capacity building so as to promote the sustainable development of OGA.
\end{abstract}

\section{Introduction}

Government data has its own uniqueness1. With the further development of OGA, the data of performance evaluation shows a trend of exponential growth. Both the resources required for the performance evaluation and the difficulty of the work increase sharply. It's harder for the third-party to make performance evaluation of OGA. There are a lot of drawbacks in the traditional performance evaluation method. Hence, the existing method needs to be improved, and the main points of improvement are listed as follows:

- The evaluators have strong subjectivity in the traditional performance evaluation of OGA. Not all the staff have rich experience of evaluation and are familiar with the development status of relevant research fields both domestic and abroad2. The scoring standards are different due to the cognitive bias of evaluators, hence the evaluation results are uncertain, which leads to the deficiencies in fairness and professionalism3.

- In the method of traditional third-party performance evaluation, the accurate ratio index is generally used to reflect the level of OGA. Fuzzy logic is a powerful tool to deal with uncertainty, nonlinearity and other ill-posedness problems4. The reasoning method of fuzzy logic is similar to the way of human thinking, which means it is suitable to express fuzzy or qualitative knowledge5. Therefore, the appropriate fuzzy processing of data can more objectively and truly reflect the level of OGA, which makes the performance evaluation more objective, justice and equity.

- The traditional third-party evaluation generally uses linear analysis method to calculate the final results. However, the linear method can only reflect the degree of OGA in general, and it can hardly dig out the hidden information in data. Nonlinear calculation is needed to reflect the complexity and fuzziness of data. Scientific evaluation system and method is very important to the sustainability of performance evaluation of OGA.

Fuzzy neural network has the advantage of fuzzy reasoning and reverse learning, it works efficiently in prediction6. By personally taking part in the third-party performance evaluation of OGA in Shandong Province, we have a deep understanding of the evaluation process and methods. Then a fuzzy neural network model based on T-S was established. The fuzzy neural network and the expert experience in third-party performance evaluation are perfectly combined in this model. Compared with the traditional third-party performance evaluation method of OGA, the evaluation method based on T-S fuzzy neural network has the advantages of high efficiency and accuracy, which can promote the development of OGA effectively.

*Corresponding author's e-mail: zhouml@sdas.org. 


\section{The development of the third-party evaluation of OGA}

\subsection{The concept of OGA and the characteristics of evaluation}

OGA refers to the full process of decision-making, implementation, management, service and results promoted by administrative organs. It can protects the public's right to know, to participate, to express and to supervise.

The performance evaluation can be used to find out whether OGA is carried out in place and whether it achieved the expectations. Government performance evaluation is the main tool to regulate the government administrative behavior and also an important way to improve the government management ability.

The index system of OGA involves a wide range of areas. OGA also has strong hierarchical characteristic. Great differences are found among the evaluation standards of governments with different levels. The evaluation data need to be compared both horizontally and vertically. There are three requirements in the evaluation of OGA. Firstly, the evaluators were required to have rich knowledge and experience with evaluation. Secondly, the scoring standards of different evaluators were required to be highly consistent. Thirdly, data must be comprehensively processed to achieve the evaluation goal. Due to the subjective differences of evaluators and the unreasonable methods used to analysis data, the evaluation results of OGA often appear to be not objective, inaccurate or contrary to the actual feelings of the public, which has an adverse effect on the sustainable development of OGA performance evaluation.

In recent years, the research on the performance evaluation of OGA mainly focus on the improvement of evaluation mode and method, but it seldom appears in the performance evaluation of OGA7. Since the evaluation model of e-government performance based on BP neural network was put forward, other evaluation models based on improved BP neural network appeared gradually. For example, e-government evaluation model based on rough set BP neural network8, e-government performance evaluation model based on PSO-BP neural network. Although BP neural network can improve the efficiency of evaluation, it has not been widely applied in the performance evaluation of OGA, which is mainly due to the lack of accuracy.

\subsection{The method and process of third-party evaluation of OGA}

The third-party evaluation institutions usually combine the quantitative data with qualitative analysis in the performance evaluation of OGA. Before the formal evaluation, the third-party evaluation institutions usually use analytic hierarchy process (AHP) to study and analyze the evaluation system existed. Combined with the actual situation of the evaluation objects, they usually construct reasonable government evaluation indicators, and assign weights to these indicators to form their own evaluation standards. The scores of quantitative data in evaluation are obtained through data capture and manual observation according to the evaluation standards, and the scores are summed to obtain the final results. Finally, based on the ranking method, comparative analysis method, average analysis method and other methods, professional evaluators analyze the data and form the performance evaluation report.

\section{Application of fuzzy neural network in third party evaluation}

\subsection{Application of T-S fuzzy neural network in evaluation of government affairs publicity}

The relationship between the performance evaluation index of OGA and the performance of government affairs publicity is not definite, but fuzzy and difficult to express accurately. Because the cognitive biases evaluators have in the standards of performance evaluation indicators, the evaluation data has strong fuzziness. Therefore, the evaluators usually rely on experience to deal with these fuzzy concepts which is difficult to describe quantitatively. Considering the superiority of fuzzy model in fuzzy knowledge expression, fuzzy theory can solve problems which are fuzzy and difficult to describe quantitatively.

Therefore, fuzzy theory and neural network can be combined to apply to the performance evaluation of OGA. The fuzzy neural network can fuzzify the input variables, and then it can carry out fuzzy reasoning9. The fuzzy neural network can process the input through the membership function, so as to carry out quantitative description. The nonlinear fitting and fuzzy expression ability of the model are improved greatly, which improves the accuracy of evaluation.

\subsection{The evaluation process of government affairs publicity performance based on T-S fuzzy neural network}

The process of performance evaluation of OGA based on T-S fuzzy neural network is shown in figure 1. Firstly, an appropriate index evaluation system was established according to the evaluation objectives of OGA. Then a fuzzy neural network structure was established based on the index evaluation system, and the fuzzy neural network was trained and tested to find the optimal parameter which is applied to the evaluation. 


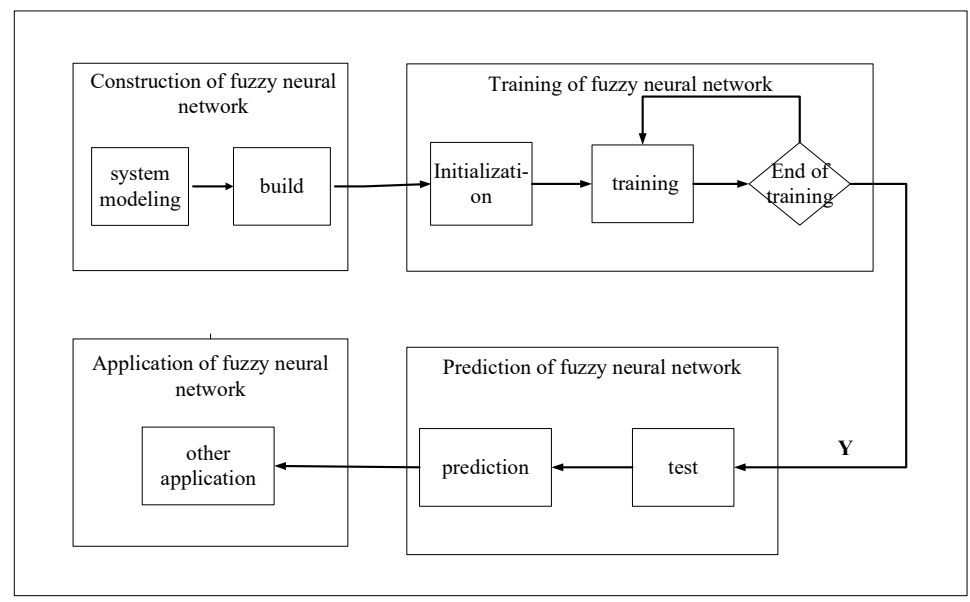

Fig 1. Performance evaluation process of government affairs publicity.

\section{Construction of third-party evaluation model}

\subsection{Performance evaluation index of open government affairs}

Taking Shandong Province as an example, the performance evaluation index of OGA is divided into five levels according to the experience of experts and the actual situation of governments. This paper uses the performance evaluation index of the third-party evaluation organization in Shandong Province to build the fuzzy neural network model, and takes the first level indexes of the evaluation system as the input. The chosen evaluation indexes are shown in table 1 .

Table1. Performance evaluation indexes of open government affairs.

$\left.\begin{array}{cc}\hline \text { First level evaluation } & \text { Weight } \\ \text { index } & \\ \hline \begin{array}{l}\text { Open operation of } \\ \text { administrative power( }\left(\mathrm{x}_{1}\right)\end{array} & (20) \\ \text { Information disclosure } \\ \text { in key areas }\left(\mathrm{x}_{2}\right)\end{array}\right)$

\subsection{T-S fuzzy neural network structure based on evaluation index}

The T-S fuzzy neural network evaluation model with four layers is built according to the performance evaluation index system of the third-party organization in Shandong Province, China. The the first layer is the input layer 10. while $x_{i}(i=1,2, \ldots, n, n$ means the number of first level evaluation index) is the input variable. In this paper, the number of first grade indicators of government affairs publicity performance evaluation is taken as the number of input variables $n=5$. There are five neural network nodes in this layer.
The second layer is the fuzzification layer, in which the input variables are fuzzified, and selects the OGA data of the third-party evaluation institutions in Shandong Province as the sample data to experiment. The score between 0 and 1 is used to evaluate the indexes of the five factors, in which 0 means the worst and 1 means the best. According to the characteristics of the evaluation of OGA, when evaluators give a zero or full marks, the score is relatively accurate, but there may be some deviation in the middle area. Hence, Gaussian function is used as the membership function to measure the membership degree of each evaluation index to the 3 levels of the OGA performance evaluation. According to the above provisions, the mathematical description can be obtained. The fuzzy subset $\operatorname{Aj}(j=1,2,3)$, which is defined on $U(U \in$ $[0,1])$, means three kind of membership functions (excellent, good and bad in OGA). $\mu A_{j}^{i}\left(x_{i}\right)$ is the membership degree of Aj to the ability of OGA of $x_{i}$. In this layer, the input variables are fuzzified according to the selected membership function to get 15 fuzzy variables, and then these fuzzy variables are sent to the fuzzy rule layer. Therefore, there are 15 neural network nodes in this layer.

The third layer is the fuzzy rule layer, and the number of nodes is the same as the number of fuzzy rules.

The fourth layer is the defuzzification layer, which is used to defuzzify the fuzzy quantity and output the performance evaluation result of OGA.

\subsection{The learning algorithm}

4.3.1 Error calculation. Where e is the error value, $y_{d}$ and $\mathrm{y}_{\mathrm{c}}$ are expected output and actual output respectively.

$$
e=\frac{1}{2}\left(y_{d}-y_{c}\right)^{2}
$$

4.3.2 Correction coefficient. Where $\mathrm{p}_{\mathrm{j}}^{\mathrm{i}}$ is the coefficient of neural network, $\alpha$ is the network learning rate, $x_{j}$ is the input variable, $\omega^{i}$ is the continuous product of the membership degree of the input variable.

$$
p_{j}^{i}(k)=p_{j}^{i}(k-1)-\alpha \frac{\partial e}{\partial p_{j}^{i}}
$$




$$
\frac{\partial e}{\partial p_{j}^{i}}=\frac{\left(y_{d}-y_{c}\right) \omega^{i}}{\sum_{i=1}^{m} \omega^{i} \cdot x_{j}}
$$

4.3.3 Parameter modification. Where $c_{j}^{i}$ and $b_{j}^{i}$ are the center and width of the membership function respectively.

$$
\begin{aligned}
& c_{j}^{i}(k)=c_{j}^{i}(k-1)-\beta \frac{\partial e}{\partial c_{j}^{i}} \\
& b_{j}^{i}(k)=b_{j}^{i}(k-1)-\beta \frac{\partial e}{\partial b_{j}^{i}}
\end{aligned}
$$

\section{Training process of neural network}

We trained the neural network by using the data of the third-party OGA evaluation of Jinan city from 2016 to 2019, Shandong Province, China. After deleting the duplicate and problem data, 1500 groups of data were randomly selected, a part of the evaluation data is shown in Table 2. The 1500 groups of data are randomly split into training data and test data according to the ratio of 5:1, and then the data is preprocessed to make it lies within the range $[-1,1]$. The values of center, width and coefficient of fuzzy membership function is modified by constantly adjusting the super parameters. The T-S fuzzy neural network is optimized repeatedly to find the best prediction model, the number of hidden layer neurons is determined to be 45 . At last, the T-S fuzzy neural network is used to test and evaluate.

Table2. Government evaluation data.

\begin{tabular}{ccccccc}
\hline Groups & $x_{1}$ & $x_{2}$ & $x_{3}$ & $x_{4}$ & $x_{5}$ & $\begin{array}{c}\text { Expected } \\
\text { value }\end{array}$ \\
\hline (1) & 20 & 30 & 10 & 20 & 20 & 100 \\
(2) & 19.46 & 30 & 10 & 19 & 20 & 98.46 \\
(3) & 18.9 & 26.63 & 9 & 20 & 20 & 94.53 \\
(4) & 14.37 & 23.86 & 10 & 20 & 20 & 88.23 \\
(5) & 14.6 & 24.83 & 9 & 18 & 20 & 86.43 \\
(6) & 10.3 & 22 & 9 & 18 & 17 & 76.3 \\
(7) & 8.99 & 14.95 & 8 & 19 & 17 & 67.94 \\
(8) & 17.75 & 28.1 & 7 & 17 & 19 & 88.85 \\
\hline
\end{tabular}

\section{Discussion}

Based on the evaluation data, the TS fuzzy neural network takes 800 iterations to achieve an acceptable error rate. As shown in figure 2, with the number of iterations increased, the prediction error rate of T-S fuzzy neural network decreases continuously. Finally, the neural network can reach the optimal goal. In addition, figure 4(a) and figure 4(b) respectively show the comparison between the expected value and the predicted value of the test data, and the absolute error of the test data. the absolute error of testing is between 0 and 0.13 , the predicted value in the test set is consistent with the actual value, and the accuracy of T-S fuzzy neural network is enough for prediction. Besides, the prediction for special data are shown in figure 3. When the data score is zero, it has an effect on the prediction result of T-S fuzzy neural network. The special data is shown in Table 3, in which both group 11 and group 13 contain zero data. The experiment is repeated as many times, and it can be seen from figure 3 that the predicted results and actual results are basically consistent. The data with zero just has little effects on the prediction results, and the results are relatively stable.

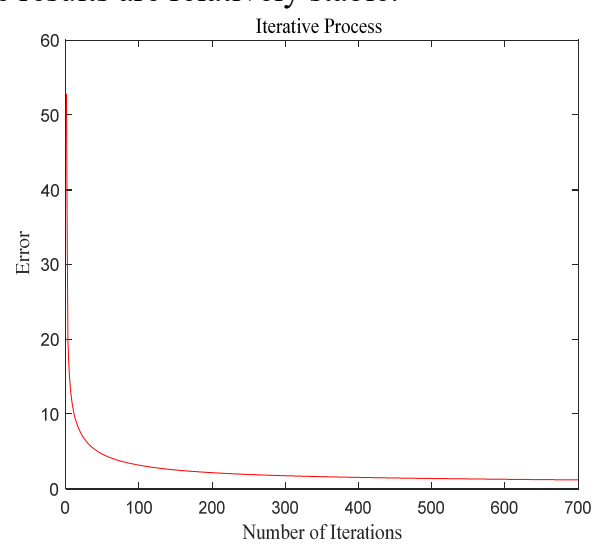

Fig 2. Iterative Process.

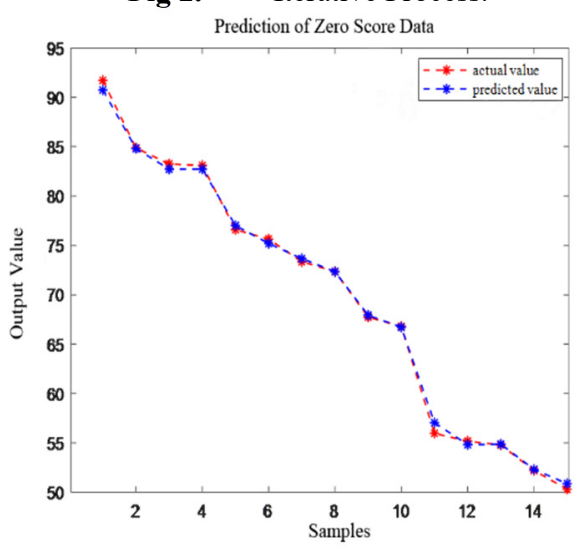

Fig 3. Prediction of zero score data. 
(a)

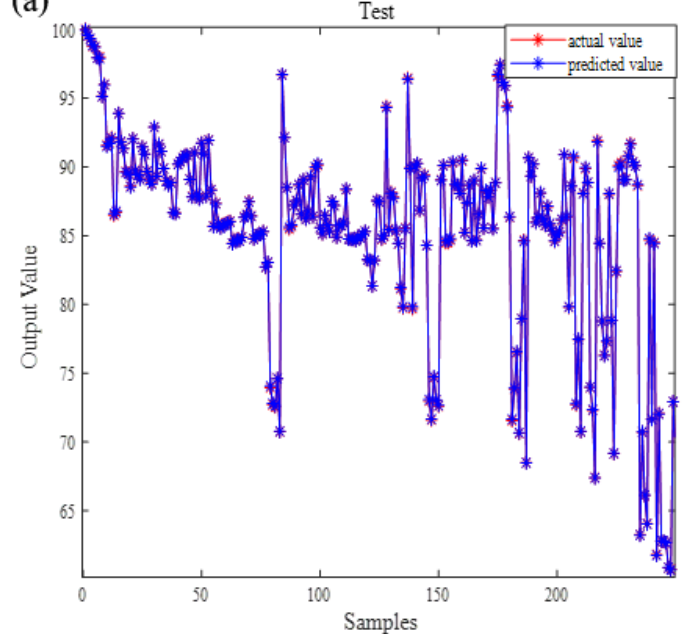

(b)

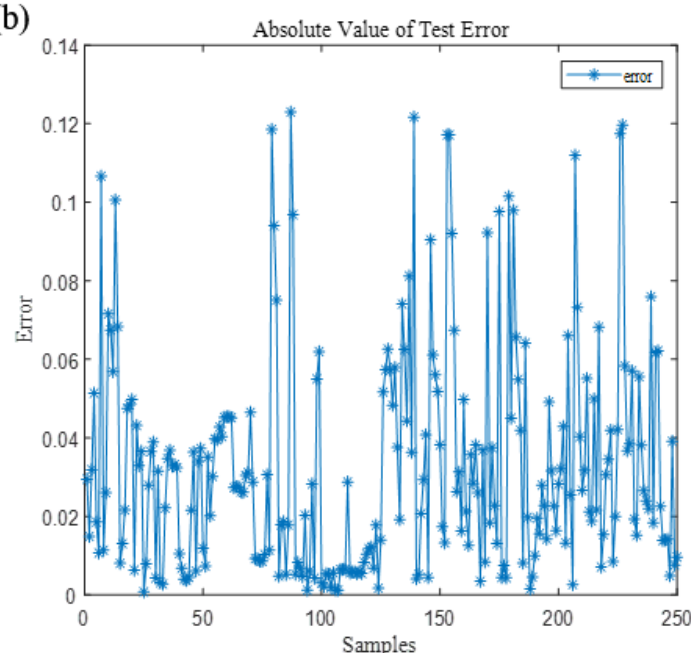

Fig 4. (a) Comparison of expected and predicted values (b) Absolute error value of test data.

Table3. Predicted results of zero score data.

\begin{tabular}{ccccccccc}
\hline Groups & $\boldsymbol{x}_{\mathbf{1}}$ & $\boldsymbol{x}_{\mathbf{2}}$ & $\boldsymbol{x}_{\mathbf{3}}$ & $\boldsymbol{x}_{\mathbf{4}}$ & $\boldsymbol{x}_{\mathbf{5}}$ & $\begin{array}{c}\text { Actual } \\
\text { value }\end{array}$ & $\begin{array}{c}\text { Expected } \\
\text { value }\end{array}$ & $\begin{array}{c}\text { Forecast } \\
\text { ranking }\end{array}$ \\
\hline 1 & 19.52 & 28.2 & 8 & 19 & 17 & 91.72 & 90.74 & 1 \\
2 & 14.33 & 27.55 & 8 & 17 & 18 & 84.88 & 84.81 & 2 \\
3 & 20 & 25.25 & 9 & 16 & 13 & 83.25 & 82.73 & 4 \\
4 & 16.4 & 24.7 & 9 & 15 & 18 & 83.1 & 82.74 & 3 \\
5 & 14.9 & 28.7 & 9 & 18 & 6 & 76.6 & 77.01 & 5 \\
6 & 20 & 24.65 & 2 & 14 & 15 & 75.65 & 75.2 & 6 \\
7 & 17.92 & 27.43 & 9 & 4 & 15 & 73.35 & 73.67 & 7 \\
8 & 8.5 & 23.85 & 9 & 19 & 12 & 72.35 & 72.34 & 8 \\
9 & 12.05 & 23.7 & 9 & 13 & 10 & 67.75 & 67.93 & 9 \\
10 & 6.85 & 17.94 & 10 & 20 & 12 & 66.79 & 66.71 & 10 \\
11 & 4.56 & 23.4 & 0 & 11 & 17 & 55.96 & 57.03 & 11 \\
12 & 16.77 & 16.4 & 6 & 8 & 8 & 55.17 & 54.82 & 13 \\
13 & 13.79 & 20 & 7 & 0 & 14 & 54.79 & 54.87 & 12 \\
14 & 13.25 & 14.95 & 5 & 9 & 10 & 52.2 & 52.31 & 14 \\
15 & 8.4 & 9.9 & 6 & 13 & 13 & 50.3 & 50.86 & 15 \\
\hline
\end{tabular}

Table4. Prediction results with the same actual scores

\begin{tabular}{ccccccccc}
\hline Groups & $\boldsymbol{x}_{\mathbf{1}}$ & $\boldsymbol{x}_{\mathbf{2}}$ & $\boldsymbol{x}_{\mathbf{3}}$ & $\boldsymbol{x}_{\mathbf{4}}$ & $\boldsymbol{x}_{\mathbf{5}}$ & $\begin{array}{c}\text { Actual } \\
\text { value }\end{array}$ & $\begin{array}{c}\text { Expected } \\
\text { value }\end{array}$ & ranking \\
\hline (1) & 13.79 & 20 & 4 & 14 & 15 & 66.79 & 68.5 & 1 \\
(2) & 14.37 & 16.42 & 9 & 9 & 18 & 66.79 & 68.2 & 2 \\
(3) & 13.25 & 20.54 & 2 & 15 & 16 & 66.79 & 67.79 & 3 \\
(4) & 14.39 & 16.4 & 4 & 15 & 17 & 66.79 & 67.66 & 4 \\
(5) & 12.36 & 17.43 & 6 & 16 & 15 & 66.79 & 67.06 & 5 \\
(6) & 6.85 & 17.94 & 10 & 20 & 12 & 66.79 & 66.84 & 6 \\
\hline
\end{tabular}

\section{Conclusions}

The results show that the government performance evaluation method based on T-S fuzzy neural network has remarkable accuracy. It can effectively solve the uncertainty problem in evaluation caused by the subjective bias of evaluators. The new model proposed in this paper is suitable for each object of evaluation, no matter district, counties or departments, and the improved evaluation method can simplify the repetitive work. Compared with the traditional evaluation method, the proposed method is more appropriate for the evaluation of OGA performance, which can reflect the complex relationship among the indicators of evaluation. The new evaluation method improves the efficiency of government performance evaluation and saves a lot of manpower, material and financial resources. It has strong advantages in the evaluation of OGA, which is meaningful for the 
sustainable development of OGA.

\section{Acknowledgments}

This work was supported by Key Research and Development Program of Shandong, China (No.2019GGX105020), Plan of Youth Innovation Team Development of colleges and universities in Shandong Province (SD2019-161) and Shandong Provincial Natural Science Foundation of China (ZR2020MG075).

\section{References}

1. Alaouie, AM. (2019) Assessing whether the design of a state forensic portal of test results complies with requirements of open government data. Forensic Science International, 1.

2. Wang, X; Dang, D; Guo, Z. (2020) Evaluating the crowd quality for subjective questions based on a Spark computing environment. Future Generation Computer Systems, 106: 426-437.

3. Fang, Yong; Mingming, Wang. (2010) Research on the Third-party Evaluation Consultation Model of National S\&T Planned Projects Based on Government Functions Transformation. In: 6th International Conference on Public Administration. Canberra. pp.136-141.

4. Mrazgua, J; Tisssir, EH; Ouahi, M. (2019) Fuzzy Fault-Tolerant H Control Approach for Nonlinear Active Suspension Systems with Actuator Failure. In:The Second International Conference on Intelligent Computing in Data Sciences.Indonesia. 148:465-474.

5. Collins, EG. (2003) Fuzzy control systems design and analysis: a linear matrix inequality approach: Kazuo Tanaka and Hua O. Wang. Automatica, 39:2011-2013.

6. Lyu, Y; Jiang, Y. (2020) Examination on avionics system fault prediction technology based on ashy neural network and fuzzy recognition. Journal of Intelligent \& Fuzzy Systems, 38:3939-3947.

7. Elavarasan, D; Vincent, DR; Sharma, V; Zomaya, AY; Srinivasan, K. (2018) Forecasting yield by integrating agrarian factors and machine learning models: A survey. Computers And Electronics In Agriculture, 155:257-282.

8. Jianli, Gao. (2020) Performance evaluation of manufacturing collaborative logistics based on BP neural network and rough set. Neural Computing \& Applications, PP. 1-16.

9. Lima-Junior, FR; Carpinetti, LCR. (2020) An adaptive network-based fuzzy inference system to supply chain performance evaluation based on SCORő metrics. Computers \& Industrial Engineering, 139.

10. Souza, P; Ponce, H; Lughofer, E. (2020) Evolving fuzzy neural hydrocarbon networks: A model based on organic compounds. Knowledge-based Systems, 203. 